\title{
Veteran Perspectives on Adaptations to a VA Residential Rehabilitation Program for Substance Use Disorders During the Novel Coronavirus Pandemic
}

\author{
Kimberly Clair ${ }^{1}$ (D) Roya ljadi-Maghsoodi ${ }^{1,2,3,4} \cdot$ Mariam Nazinyan $^{2,3} \cdot$ Sonya Gabrielian ${ }^{1,2,3}$. \\ Ippolytos Kalofonos ${ }^{1,2,3,5,6}$
}

Received: 1 November 2020 / Accepted: 23 February 2021 / Published online: 6 March 2021

(c) This is a U.S. government work and not under copyright protection in the U.S.; foreign copyright protection may apply 2021

\begin{abstract}
Individuals attending residential rehabilitation programs for substance misuse are particularly vulnerable to treatment disruptions spurred by the novel coronavirus disease 2019 (COVID-19) pandemic. We describe adaptations to services within a large residential rehabilitation program for under-resourced veterans, report veterans' experiences with these changes, and outline successes and challenges encountered throughout adjustment to the pandemic. Data collected from two focus groups with nine veterans engaged in this program during the pandemic highlight experiences of inconsistent communication about residential policies, interruptions to medical and addiction services, and feelings of confinement and social isolation. Overall, these findings suggest the need for health systems to support clients in taking an active role in communications, provide additional technical and social support in transitioning to virtual health services, and offer alternative means for clients to maintain social connection during a pandemic. Understanding clients' perspectives can inform strategies to promote continuity of care and enhanced care experiences.
\end{abstract}

\section{Introduction}

Kimberly Clair

k.clair@ucla.edu

1 Department of Veterans Affairs, Greater Los Angeles Healthcare System, 11301 Wilshire Blvd, Bldg 210A, Los Angeles, CA 90073, USA

2 Department of Psychiatry and Biobehavioral Sciences, UCLA David Geffen School of Medicine, Jane and Terry Semel Institute for Neuroscience and Human Behavior, 760 Westwood Plaza, Los Angeles, CA 90095, USA

3 Center of Excellence for Veteran Resilience and Recovery, UCLA, VA Greater Los Angeles Healthcare System, 11301 Wilshire Boulevard, Los Angeles, CA 90073, USA

4 UCLA Division of Population Behavioral Health, Jane and Terry Semel Institute for Neuroscience and Human Behavior, Los Angeles, CA, USA

5 UCLA Center for Social Medicine and Humanities, Jane and Terry Semel Institute for Neuroscience and Human Behavior, 760 Westwood Plaza, Los Angeles, CA 90095, USA

6 UCLA International Institute, 1248 Bunche Hall, Los Angeles, CA 90095, USA
The novel coronavirus (COVID-19) pandemic necessitated rapid adaptations to providing medical, mental health and substance use disorder (SUD) services (Arevian et al., 2020). These changes-including guidelines for physical distancing and transitions to virtual care modalities - are particularly disruptive for individuals with mental illness and SUD who have experienced homelessness ("homeless-experienced persons"). This vulnerable group is disproportionally burdened by the COVID-19 pandemic; many homeless-experienced persons are highly reliant on continuous access to medications, support groups, and in-person services from trusted providers (Kavoor, 2020; Lin et al., 2020).

Regardless of housing status, many persons with mental illness have experienced exacerbations of psychiatric symptoms (e.g., anxiety or depression) as a result of the COVID-19 pandemic; in particular, physical distancing measures may increase loneliness, isolation, and suicide risk (Druss, 2020; Holt-Lunstad et al., 2015; Pfefferbaum \& North, 2020). Many individuals with SUD, regardless of co-occurring mental illness, have experienced increased urges for substances as a result of stress and uncertainty associated with the pandemic, while stigma towards this 
population may cause healthcare providers to de-prioritize their needs (Marsden et al., 2020; Volkow, 2020). Moreover, homeless-experienced persons are highly vulnerable to epidemics, with disease transmission facilitated by congregate living in shelters, transitional housing, and encampments; high rates of chronic medical illness, psychiatric problems, and SUD; and reduced access to protections, e.g., handwashing stations and masks (Tsai \& Wilson, 2020). Recognizing these vulnerabilities and aiming to curb COVID-19 transmission, health systems have rapidly adopted virtual care modalities to substitute for in-person services (Heyworth et al., 2020). This shift may be particularly disruptive for homeless-experienced persons attending residential rehabilitation programs-programs that provide rehabilitative and/or clinical care in a residential setting-for behavioral health and SUD, where regular group meetings and social connections to peers are integral to recovery (Samuels et al., 2020; Volkow, 2020).

Residential treatment programs for people experiencing homelessness face unique challenges addressing the needs of clients-many of whom have co-occurring mental illness and SUD-while rapidly implementing new policies and care modalities to mitigate the spread of COVID-19. Understanding clients' experiences with these changes can illuminate specific strategies that facilitate service engagement and lessen psychosocial distress for this vulnerable population during this challenging time. To inform programs and services for this highly vulnerable population, this paper describes: (1) the response to the COVID-19 pandemic by a large residential rehabilitation program serving homeless-experienced veterans, (2) the voiced experiences of homeless-experienced veterans engaged in residential treatment for behavioral health issues and SUD shortly after the COVID-19 pandemic began, and (3) successes and challenges the program encountered throughout active quality improvement efforts.

The Department of Veterans Affairs (VA) is the nation's largest provider of residential rehabilitation services for homeless persons and a valuable setting to examine the responses of homeless-experienced clients to COVID-19-related care adaptations. The VA and its community partners provide clinical rehabilitation and treatment services for veterans with multiple and severe conditions, including mental illness, SUD, and/or psychosocial deficits (U.S. Department of Veterans Affairs [USDVA], 2020). We describe the response of a residential rehabilitation program located at a large VA medical center in Southern California; the program has nearly 300 beds and its affiliated VA serves more homeless veterans than any VA in the nation.

In mid-March, coinciding with the "Safer at Home" lockdown measures instituted by the city mayor, this residential rehabilitation program rapidly responded to established national guidelines for COVID-19 public health precautions by mandating COVID-19 testing at admission, instituting daily screening for COVID-19 symptoms for veterans and staff, implementing physical distancing policies for treatment groups and recreational activities, and transitioning inperson care to phone or virtual services (audio and/or video). The program also reduced admissions in order to set aside quarantine beds for veterans with pending COVID-19 test results or homeless veterans who were COVID-19 positive and asymptomatic or symptomatic but suitable for ambulatory care. In the initial phases of this transition, the program also implemented a no-visitor policy for veterans and limited recreational activities to outdoor settings with fewer participants and staff supervision. Psychosocial treatment, psychotherapy, and psychiatric care were provided through virtual modalities; some group therapy was performed inperson, though restricted to fewer than 10 participants and held outdoors to facilitate physical distancing. Primary care was offered in-person or through virtual modalities, depending on the patient complaint and clinician triage of that complaint. Veterans received meals delivered to their rooms and were instructed to wear face coverings when outside their room and practice frequent hand-washing. To keep veterans informed of evolving policy changes, the program provided flyers, weekly town-halls (by telephone), and updates within veteran-led community meetings.

These policies and changes in service provision evolved over the course of the pandemic; for example, the program adopted routine surveillance testing for veterans and staff and additional protocols were developed to mitigate the spread of disease. Though the initial changes to program services reflected established national guidelines (Centers for Disease Control and Prevention [CDCP], 2020), few have explored client perspectives on and experiences with these changes.

\section{Methods}

To fill this knowledge gap and inform services and policies for homeless-experienced veterans receiving residential treatment, we recruited a convenience sample of nine participants of the aforementioned residential rehabilitation program who were associated with an established veteran stakeholder group (UCLA/VA Center of Excellence on Veteran Resilience and Recovery's Veteran Engagement Group) that provides input on quality improvement and research activities related to homeless veterans with behavioral health concerns. We were limited to nine participants by the Paperwork Reduction Act of 1995, which limits data collection on quality improvement efforts to fewer than ten veterans without additional approvals (Paperwork Reduction Act of 1995). We conducted two telephonebased focus groups (one with five veterans, another with 
four), audio-recorded and transcribed responses, and took detailed field notes during the sessions. The focus group sessions took place in early May, approximately two months after COVID-19 public health precautions took effect at the residential rehabilitation program and were led by a health services researcher experienced in qualitative interviewing (KC), with support from four other members of the research team-health services researchers and psychiatrists with qualitative expertise (IK, SG, MN, RI-M). We selected focus groups as our method of data collection because we were interested in participants' perceptions, ideas, thoughts, and opinions (Krueger \& Casey, 2000). We also wanted participants to hear one another's experiences, which may "cue" other participants to share information they may not have provided through surveys or one-on-one interviews (Morgan \& Krueger, 1993). Further, qualitative interviews can highlight the needs of highly vulnerable populations (Israel et al., 2005). Our focus groups were phone-based due to COVID-19-related physical distancing precautions. Phone-based focus groups have been shown to produce data of similar quality to those obtained through face-to-face focus groups and may facilitate recruitment and participants' disclosure of sensitive experiences (Frazier et al., 2010). Participants were reimbursed with an Amazon e-card valued at \$50.

Facilitators utilized a semi-structured guide that inquired about participants' experiences with housing, mental health, and medical health services, including changes in access to and perceived quality of care. For example, participants were asked whether they experienced any changes with respect to making medical appointments and whether they had any concerns about the amount or type of care they received. All researchers took verbatim notes during the focus groups sessions. These notes were compiled and analyzed inductively using constant comparison analysis to identify similarities and differences across participants' experiences (Onwuegbuzie et al., 2009; Strauss \& Corbin, 1998). Five members of the research team reviewed the transcripts independently to identify preliminary themes. Codes were then discussed among the five team members and a codebook was developed by two authors (KC and IK) through iterative discussion that focused on specific challenges participants experienced; changes in their relationships with staff, medical providers, and friends/family; and recommendations to improve care during the COVID-19 pandemic. All discrepancies were resolved by two authors (KC and IK). Two authors ( $\mathrm{KC}$ and $\mathrm{MN}$ ) coded the verbatim notes by applying one or more relevant codes from the codebook to the notes; the notes were then grouped by code for analysis. Participants' electronic medical records were reviewed following completion of the phone-based focus groups to obtain participants' demographic characteristics (gender, race, ethnicity), mental health diagnoses, and SUD.
We also present the program's response to veterans' concerns. Key findings from the focus group were collected simultaneously to process improvement efforts to balance veteran needs and public health precautions. We assured that process improvement efforts addressed our key findings. Program responses were compiled by reviewing all facility- and program-level written correspondence to staff during the pandemic. We extracted information about policy changes that pertained to findings highlighted in our qualitative data collection; three authors (KC, RI-M, SG) iteratively discussed and achieved consensus regarding the successes and obstacles of these responses.

The authors report no potential conflicts of interest with respect to the research, authorship, and/or publication of this article. All authors certify their responsibility for the manuscript. This project was formally approved as a quality improvement project by the VA Greater Los Angeles IRB Administrator.

\section{Results}

From the focus groups, three major areas in which participants experienced challenges adapting to COVID-19-related changes were identified: communication, interruption of services, and social isolation/confinement (Table 1).

\section{Communication}

Many participants expressed confusion about COVID19-related changes to policies and protocols in the residential rehabilitation program. While some mentioned a lack of access to information, attributed to not having internet or television in their rooms, others described receiving mixed or inconsistent messages from program staff. Specifically, participants expressed confusion about accessing outdoor spaces, COVID-19 testing procedures, meal delivery, and mask-wearing. One participant felt that "the system changed day to day" depending on which staff member was on duty. Another felt that the shifts in regulations were applied unevenly and that staff were "picking and choosing" who could access outdoor spaces or communal areas. One participant who contemplated discontinuing his treatment explained, "we didn't get a sense of why this was happening" and decided to seek information outside of the VA.

Participants also perceived a disconnect between the policies they were instructed to follow and behaviors they observed among staff and members of the general community. Several participants did not understand why they had restricted outdoor access while community members walked freely around the medical center campus, sometimes without masks. This prompted a discussion of "double standards," with many participants feeling that others on the VA campus 
Table 1 Recommendations proposed by focus group participants, May $2020(N=9)$

\begin{tabular}{|c|c|}
\hline \multicolumn{2}{|l|}{ Communication } \\
\hline Provide daily updates & "I wish they had said hey it's Day 13 of Covid here's what we are doing" \\
\hline Create a standardized messaging system & "Have one communication board, one source of accurate information" \\
\hline Give residents an active role & $\begin{array}{l}\text { "Staff has a huddle in the morning-do the same with patients to communicate the infor- } \\
\text { mation they got earlier from others" }\end{array}$ \\
\hline Explain rationale for policy changes & $\begin{array}{l}\text { "When we read stuff from outside sources, we jump to conclusions, best to get everyone } \\
\text { together, say this is what's going on and why" }\end{array}$ \\
\hline Explain COVID-19 testing procedures & $\begin{array}{l}\text { "Would be nice to know the procedure [for COVID] testing. Someone was bleeding } \\
\text { in line and that gave him and others anxiety. Preparing veterans for the test would be } \\
\text { helpful" }\end{array}$ \\
\hline \multicolumn{2}{|l|}{ Interruption of Services } \\
\hline Provide technical support for clients & $\begin{array}{l}\text { "A liaison could help for people who don't have phones, don't know how to use technol- } \\
\text { ogy" }\end{array}$ \\
\hline Provide training for staff & $\begin{array}{l}\text { "There needs to be more training for these people [who] keep us safe...they need to be } \\
\text { properly trained for what Veterans are going through" }\end{array}$ \\
\hline \multicolumn{2}{|l|}{ Confinement/Social Isolation } \\
\hline Encourage participation in recreational activities & $\begin{array}{l}\text { "The most helpful stress reliever has been recreational activities...Certain staff at the rec } \\
\text { center were helpful getting people [to go] out" }\end{array}$ \\
\hline Provide alternative resources for social engagement & $\begin{array}{l}\text { "If [only] there had been some kind of staff involvement in terms of how to find other } \\
\text { support, like Zoom" }\end{array}$ \\
\hline
\end{tabular}

did not have to follow the same regulations that they had to follow. Several participants believed that testing procedures differed for staff and for residents. For example, two participants believed that testing for staff was not mandatory, putting residents in danger. Another felt that staff were tested much later than residents and did not understand why COVID-19 testing procedures for staff and residents did not take place at the same time. To prevent confusion, participants expressed a desire to be included in communications and to receive consistent messaging across sources. One participant commented, "I wish [staff] would ask us for suggestions or act like we had a voice, like they were in it with us, not protecting us."

The residential rehabilitation program responded to these concerns by expanding opportunities for communication with residents. As the pandemic progressed, the program communicated policy changes both through written documentation and in community meetings. The program instituted an "Ask Your Doctors" forum and established an open-door policy with leadership and clinicians for veterans to ask questions and express concerns. Expanding channels of communication with residents was critical as the program responded to surges of positive cases at the facility and community levels with iterative policy changes. Moreover, the program established a procedure for weekly COVID-19 surveillance among staff and prioritized all staff for COVID-19 vaccination. Though these efforts aimed to provide transparent and open communication-while minimizing risks to residents and staff - the evolving nature of the pandemic often necessitated dynamic and rapid policy change. At times, residents were not able to voice their concerns or contribute to policy recommendations; the severity of community spread simply necessitated top-down policy changes that were consistent across the facility and its programs.

\section{Interruption of Services}

We asked participants to describe how COVID-19 impacted their experiences with healthcare and substance use treatment services. Participants expressed frustration about being able to access routine medical and SUD treatment services, with a general perception that all medical appointments had been cancelled and all recovery programs ceased. One participant conveyed a sense of abandonment in response to the disruption in medical services, noting "all the staff with letters behind their names left." An older male participant felt unsafe visiting a hospital or other medical facility during the pandemic and resolved to wait "until it gets back to normal" to resume care. Several participants had difficulty making appointments in specialty care (at the main medical center). One woman felt "cut off" from her Rheumatology and Pulmonology specialists; another had trouble making appointments in Gastroenterology.

The abrupt transition from in-person SUD groups to virtual or small-group meetings also proved difficult for participants. One participant who was highly motivated to start treatment for his substance use disorder but entered the facility shortly before public health precautions took place, described, "As far as recovery goes, I struck out." Many participants were confused as to why they couldn't continue inperson meetings with either medical providers or substance use groups as long as they adhered to physical distancing. 
Respondents had mixed feelings about the virtual care offered by the medical center: for some, primary care or specialty medical appointments via video felt impersonal or "didn't make any sense." For others, it was "better than nothing." A few participants did not have logistical difficulties connecting with social workers, medical providers, or psychologists through virtual modalities and in rare cases, participants spoke positively of the transition. Participants suggested providing technical instruction or assistance to improve adoption of virtual care for elderly clients and others who struggled with this technology and training for staff so they could provide such support.

While the abrupt transition to virtual services presented initial challenges, the facility was able to institute a mix of face-to-face and virtual (video and telephone) care as the pandemic progressed; this change was the result of routinized surveillance, screening, and testing procedures, as well as the enactment of policies to address key public health precautions (including universal masking and eye protection). Outdoor SUD groups (with masks and physical distancing) with limited attendance became routinely implemented within the program. Within the program and the facility, nurses were used to triage medical concerns and facilitate the appropriate modality of care to address veterans' needs. Though the increased availability of face-to-face care for both medical needs and psychosocial rehabilitation responded to participants' key concerns, there remains a "digital divide" (Lindsay et al., 2007) across socioeconomic strata (i.e., homeless-experienced veterans are less likely to use health information technology) and disparities in virtual care use plague certain under-resourced groups. For example, although the VA began offering a "digital divide" consult to provide iPads to Veterans in need of telehealth capabilities, disparities in accessing and utilizing virtual care persisted. Adoption of video care among veterans with serious mental illness (which is highly associated with homelessness) during the pandemic was less than that among veterans with other mental health diagnoses (Raja et al., 2021); a portion of vulnerable veterans in the program likely continued to suffer from service interruption despite concerted efforts to enhance access.

\section{Confinement and Social Isolation}

Adapting to physical distancing measures was particularly challenging for participants. Nearly all participants described feelings of anger, isolation, and loneliness associated with their restricted mobility, noting that they were "totally isolated from the rest of the world," and that being confined to their rooms felt like they were imprisoned or being punished. Since participants were tested early on for COVID-19 for admission into the rehabilitation program, many did not understand why they couldn't continue to congregate with peers who had also tested negative. Not being allowed to see loved ones was difficult for many participants, though some successfully turned to phone and video chat as a substitute for social connection.

Despite these challenges, some participants suggested that these precautions made them feel safer and that being "completely cut off from all substances" may help with recovery. Others found they had more time for self-reflection and were able to gain confidence in their own abilities to get through difficult times. One participant remarked: "When I first came here, I didn't think I could fix myself, but then I had to start giving myself more positive self-talk, and started to rely on my inner strengths more." Another participant felt that the added restrictions encouraged him to be pro-active about his recovery: "I think it made me stronger, even with the deprivation." Participants mentioned recreational activities, such as walks, yoga classes, and spending time outdoors as important ways to release stress. However, not all participants were aware of virtual opportunities available to them to take part in treatment. For example, several participants discussed using video conferencing software to participate in social or community-based events (e.g., 12-step meetings), which surprised another participant who wished he had known about this resource.

Throughout the VA, several practices were implemented to address social isolation, including efforts to improve veterans' technological literacy and increase opportunities for safe interpersonal connections. For example, a policy was put into effect to provide smartphones to every homeless veteran at the facility who lacked a smartphone; moreover, individual coaching was offered to veterans who struggled to use video conferencing platforms on their smartphones or other devices. Though these policies aimed to address disparities in technology access and use-and helped many veterans-a distinct group of veterans continued to lack the digital competency to use smartphones without more personalized training.

Moreover, a pilot program was established to open breakrooms to residents while recreational therapy increased their offerings of scheduled outdoor activities, including campus walks and yoga. These efforts clearly responded to participants' feedback; however, regardless, many residents continued to feel confined by the strict restrictions on outdoor times and recreational activities necessitated by the pandemic and its public health precautions.

\section{Discussion}

Though derived from a small convenience sample of veterans from a single VA residential rehabilitation program, these voiced findings from veterans provide a glimpse into the experiences of a highly vulnerable population in a region 
of the country that shut down early in the pandemic. These focus groups highlight ways to improve the experiences of individuals in residential rehabilitation programs for substance misuse during this pandemic, particularly underresourced clients, such as those experiencing homelessness.

First, clients depend on clear and consistent communication of COVID-19-related policies and the underlying rationale for these policies. Focus group participants highlighted inconsistencies in COVID-19 communication and felt that they received mixed messages from program staff. Although at the time we conducted our focus groups, this residential rehabilitation program was still in the process of developing a messaging system, the confusion participants expressed regarding COVID-19-related policies-including their ability to access to outdoor spaces, visitors, and common areas-suggests the need for greater consistency from staff and a plan for effectively communicating policy changes with residents. While studies show that effective and rapid communication is critical for individuals experiencing quarantine (Brooks et al., 2020), giving clients an active role in communication efforts, such as asking them directly for suggestions, may alleviate confusion and promote self-efficacy, particularly during disasters (Banerjee, 2020; Matthieu \& Carbone, 2020; Steffen \& Fothergill, 2009). The residential rehabilitation program's "Ask Your Doctors" forum and open-door policy with leadership represent one such effort to include residents in communication and respond directly to their concerns.

Second, the disruption to medical and SUD services left many clients feeling completely cut off from care. Clients receiving residential services for SUD expect to receive inperson group meetings and psychosocial support, which are among the best practices for rehabilitation treatment (de Andrade et al., 2019). Although systematic reviews have identified feasibility and acceptability of telemedicine among individuals with mental illness and/or SUD (LawesWickwar et al., 2018; Young, 2012), providing additional technical and social support for residents unaccustomed to using virtual care platforms may help with their adjustment (Kavoor, 2020). Particularly as health systems scramble to convert services to virtual modalities, there may be value in offering alternative resources and support groups suited to clients' needs, motivation, and past experiences (Donovan et al., 2013). However, not all clients will want to engage with virtual care. Effective treatment for patients with SUD, who are likely to face added barriers accessing telemedicine, should emphasize flexibility and patient choice (Shakir \& Wakeman, 2020).

Finally, these findings highlight the importance of continued social connection for individuals in residential rehabilitation, for whom extended periods of confinement and restricted mobility can lead to feeling punished and exacerbate existing mental health symptoms (Galea et al., 2020;
Merchant \& Lurie, 2020). Participants emphasized the value of participating in outdoor activities and finding alternative mechanisms for social connection (such as Zoom meetings) to cope with feelings of confinement and isolation; there are likely benefits of keeping clients informed of alternative avenues for social engagement and facilitating access, whenever possible, to virtual peer-led meetings and support groups.

Residential rehabilitation programs for individuals with SUD face unique challenges responding to clients' needs during a pandemic. Understanding their experiences and perspectives can inform future efforts to promote continued connection to high-quality care for this vulnerable population and may be useful for other vulnerable populations experiencing rapid adaptations to services.

Author Contributions All authors certify their responsibility for the manuscript. KC contributed to the study's design, data collection, analysis and interpretation of results, and writing of this manuscript. RI-M contributed to the study's conception, design, data collection, interpretation of results, and writing of this manuscript. MN contributed to the study's data collection, analysis and interpretation of results. SG contributed to the study's conception, design, data collection, interpretation of results, and writing of this manuscript. IK contributed to the study's conception, design, data collection, interpretation of results, and writing of this manuscript.

Funding This work was supported by the UCLA/VA Center of Excellence (COE) on Veteran Resilience and Recovery. Dr. Ijadi-Maghsoodi is supported in part by the National Institute on Drug Abuse of the National Institutes of Health under Award Number K12DA000357. Dr. Gabrielian is supported in part by VA HSR\&D Career Development Award 15-074. Dr. Kalofonos is supported in part by the Department of Health and Human Services/Agency for Healthcare Research and Quality under award number 1K12HS026407-01 and is supported by the American Council of Learned Societies. These funding agencies were not involved in the research or authorship of the manuscript.

Data Availability This material has not been published and is not under consideration for publication elsewhere. These data have not been published previously nor accepted for publication.

\section{Declarations}

Conflict of interest The authors gave no relevant financial or non-financial interests to disclose.

Ethical Approval This project was formally approved as quality improvement by the VA Greater Los Angeles IRB Administrator.

Informed Consent Informed consent was obtained from all individual participants included in the study. Verbal informed consent was obtained from all participants prior to the focus groups.

\section{References}

Arevian, A. C., Jones, F., Moore, E. M., Goodsmith, N., Aguilar-Gaxiola, S., Ewing, T., et al. (2020). Mental health community and 
health system issues in COVID-19: Lessons from academic, community, provider and policy stakeholders. Ethnicity \& Disease, 30(4), 695-700. https://doi.org/10.18865/ed.30.4.695

Banerjee, D. (2020). The COVID-19 outbreak: Crucial role the psychiatrists can play. Asian Journal of Psychiatry, 50, 102014. https ://doi.org/10.1016/j.ajp.2020.102014

Brooks, S. K., Webster, R. K., Smith, L. E., Woodland, L., Wessely, S., Greenberg, N., et al. (2020). The psychological impact of quarantine and how to reduce it: Rapid review of the evidence. The Lancet, 395(10227), 912-920. https://doi.org/10.1016/S0140 $-6736(20) 30460-8$

Centers for Disease Control and Prevention. (2020, May 1). Ten ways healthcare systems can operate effectively during the COVID-19 pandemic. Retrieved October 29, 2020, from https://www.cdc. gov/coronavirus/2019-ncov/hcp/ways-operate-effectively.html

de Andrade, D., Elphinston, R. A., Quinn, C., Allan, J., \& Hides, L. (2019). The effectiveness of residential treatment services for individuals with substance use disorders: A systematic review. Drug and Alcohol Dependence, 201, 227-235. https://doi.org/10.1016/j. drugalcdep.2019.03.031

Donovan, D. M., Ingalsbe, M. H., Benbow, J., \& Daley, D. C. (2013). 12-Step interventions and mutual support programs for substance use disorders: An overview. Social Work in Public Health, $28(3$ 4), 313-332. https://doi.org/10.1080/19371918.2013.774663

Druss, B. G. (2020). Addressing the COVID-19 pandemic in populations with serious mental illness. JAMA Psychiatry, 77(9), 891892. https://doi.org/10.1001/jamapsychiatry.2020.0894

Frazier, L. M., Miller, V. A., Horbelt, D. V., Delmore, J. E., Miller, B. E., \& Paschal, A. M. (2010). Comparison of focus groups on cancer and employment conducted face to face or by telephone. Qualitative Health Research, 20(5), 617-627. https://doi. org/10.1177/1049732310361466

Galea, S., Merchant, R. M., \& Lurie, N. (2020). The mental health consequences of COVID-19 and physical distancing: The need for prevention and early intervention. JAMA Internal Medicine, 180(6), 817-818. https://doi.org/10.1001/jamaintern med.2020.1562

Heyworth, L., Kirsh, S., Zulman, D., \& Kizer, K. W. (2020). Expanding access through virtual care: The VA's early experience with Covid-19. NEJM Catalyst. https://doi.org/10.1056/CAT.20.0327

Holt-Lunstad, J., Smith, T. B., Baker, M., Harris, T., \& Stephenson, D. (2015). Loneliness and social isolation as risk factors for mortality: A meta-analytic review. Perspectives on Psychological Science, 10(2), 227-237. https://doi.org/10.1177/1745691614568352

Israel, B. A., Eng, E., Schulz, A. J., \& Parker, A. E. (Eds.). (2005). Methods in community-based participatory research for health. Jossey-Bass.

Kavoor, A. R. (2020). COVID-19 in people with mental illness: Challenges and vulnerabilities. Asian Journal of Psychiatry, 51, 102051. https://doi.org/10.1016/j.ajp.2020.102051

Krueger, R. A., \& Casey, M. A. (2000). Focus groups: A practical guide for applied researchers (3rd ed.). Sage.

Lawes-Wickwar, S., McBain, H., \& Mulligan, K. (2018). Application and effectiveness of telehealth to support severe mental illness management: Systematic review. JMIR Mental Health, 5(4), e62. https://doi.org/10.2196/mental.8816

Lin, L., Fernandez, A. C., \& Bonar, E. E. (2020). Telehealth for substance-using populations in the age of coronavirus disease 2019: Recommendations to enhance adoption. JAMA Psychiatry. https ://doi.org/10.1001/jamapsychiatry.2020.1698

Lindsay, S., Smith, S., Bell, F., \& Bellaby, P. (2007). Tackling the digital divide: Exploring the impact of ICT on managing heart conditions in a deprived area. Information, Communication \& Society, 10(1), 95-114. https://doi.org/10.1080/13691180701193127
Marsden, J., Darke, S., Hall, W., Hickman, M., Holmes, J., Humphreys, K., et al. (2020). Mitigating and learning from the impact of COVID-19 infection on addictive disorders. Addiction, 115(6), 1007-1010. https://doi.org/10.1111/add.15080

Matthieu, M. M., \& Carbone, J. T. (2020). Collective action among US veterans: Understanding the importance of self-efficacy, collective efficacy, and social support. Journal of Community Psychology, 48, 1985-1986. https://doi.org/10.1002/jcop.22397

Merchant, R. M., \& Lurie, N. (2020). Social media and emergency preparedness in response to novel coronavirus. JAMA, 323(20), 2011-2012. https://doi.org/10.1001/jama.2020.4469

Morgan, D., \& Krueger, R. (1993). When to use focus groups and why. In D. L. Morgan (Ed.), Successful focus groups: Advancing the state of the art (pp. 3-19). SAGE Publications Inc. https://doi. org/10.4135/9781483349008

Onwuegbuzie, A. J., Dickinson, W. B., Leech, N. L., \& Zoran, A. G. (2009). A qualitative framework for collecting and analyzing data in focus group research. International Journal of Qualitative Methods, 8(3), 1-21. https://doi.org/10.1177/1609406909 00800301

Paperwork Reduction Act of 1995, 5 C.F.R. §1320, 2020.

Pfefferbaum, B., \& North, C. S. (2020). Mental health and the Covid19 pandemic. New England Journal of Medicine, 383, 510-512. https://doi.org/10.1056/NEJMp2008017

Raja, P. V., Gabrielian, S., \& Doran, N. (2021). Access to care for veterans with serious mental illness during the COVID-19 pandemic. Psychiatric Services. https://doi.org/10.1176/appi.ps.202000898

Samuels, E. A., Clark, S. A., Wunsch, C., Jordison Keeler, L. A., Reddy, N., Vanjani, R., et al. (2020). Innovation during COVID19: Improving addiction treatment access. Journal of Addiction Medicine, 14(4), e8-e9. https://doi.org/10.1097/ADM.00000 00000000685

Shakir, M., \& Wakeman, S. (2020). Substance use disorder and telemedicine: Opportunity and concern for the future. Journal of General Internal Medicine. https://doi.org/10.1007/s11606-02006299-8

Steffen, S. L., \& Fothergill, A. (2009). 9/11 volunteerism: A pathway to personal healing and community engagement. The Social Science Journal, 46(1), 29-46. https://doi.org/10.1016/j.sosci j.2008.12.005

Strauss, A., \& Corbin, J. (1998). Basics of qualitative research: Techniques and procedures for developing grounded theory. Sage.

Tsai, J., \& Wilson, M. (2020). COVID-19: a potential public health problem for homeless populations. The Lancet Public Health, 5(4), e186-e187. https://doi.org/10.1016/S2468-2667(20)30053-0

U.S. Department of Veterans Affairs. Domiciliary Care for Homeless Veterans Program-Veterans Experiencing Homelessness [General Information]. Retrieved October 19, 2020 from https://www. va.gov/homeless/dchv.asp

Volkow, N. D. (2020). Collision of the COVID-19 and addiction epidemics. Annals of Internal Medicine, 173(1), 61-62. https://doi. org/10.7326/M20-1212

Young, L. B. (2012). Telemedicine interventions for substance-use disorder: A literature review. Journal of Telemedicine and Telecare, 18(1), 47-53. https://doi.org/10.1258/jtt.2011.110608

Publisher's Note Springer Nature remains neutral with regard to jurisdictional claims in published maps and institutional affiliations. 